\title{
Relação hepatosomática e esplenosomática em peixes teleósteos de cultivo intensivo
}

\author{
Marcos Tavares-Dias ${ }^{1,3}$ \\ Maurício L. Martins ${ }^{1}$ \\ Flávio R. Moraes ${ }^{1,2}$
}

\begin{abstract}
Hepatosomatic and splenosomatic relation of intensively cultivated teleost fishes. The mean values of hepatosomatic relation (HSR), splenosomatic relation (SSR) and relative condition factor $(\mathrm{Kn})$ in Nile tilapia (Oreochromis niloticus Trewavas, 1983); pacu (Piaractus mesopotamicus Holmberg, 1887); piauçu (Leporinus macrocephalus Garavello \& Britski, 1988) and tambacu hybrid ( $P$. mesopotamicus male x Colossoma macropomum Cuvier, 1818 female) are described. The experiment was carried out from feefishing farm situated in Franca, São Paulo State, Brazil. Nile tilapia showed the greatest HSR compared to pacu, piauçu and tambacu. Although, in the last three fishes the relation was similar. The SSR between tilapia and piausu was similar, but smaller than observed in pacu and tambacu. Nevertheless, SSR in pacu and tambacu was different. The hepatic weight/body weigth relation and hepatic weight/body length relation presented positive correlation in all studied fishes. The splenic weight/body weigth relation and splenic weight/body length relation were not significant $(\mathrm{P}>0.05)$ in Nile tilapia, pacu and tambacu, but highly significant in piauçu $(\mathrm{P}<0.01)$.

KEY WORDS. Hepatosomatic relation, Splenosomatic relation, Oreochromis niloticus, Piaractus mesopotamicus, Leporinus macrocephalus, tambacu hybrid
\end{abstract}

Os peixes podem estocar grande quantidade de gordura e glicogênio no fígado (HOAR \& RANDALl 1971). O fígado dos Characidae Colossoma macropomum Cuvier, 1818 e Piaractus mesopotamicus Holmberg, 1887 é o principal sítio de armazenamento de glicogênio e certa quantidade de gordura também pode ser estocada nesse órgão (SAINT-PAUL 1984; OlivEIRA et al. 1997a,b). Entretanto, esse teor de gordura parece ser menor que o observado no curimbatá Prochilodus scrofa Steindachner, 1881 (Prochilodontidae) (VIEIRA 1984). Variações na quantidade de gordura e/ou glicogênio estocados no fígado influenciam significativamente no peso desse órgão (HEIDINGER \& CRAWFORD 1977), alterando assim a relação hepatosomática.

1) Laboratório de Patologia de Organismos Aquáticos, Centro de Aqüicultura da Universidade Estadual Paulista. Via de Acesso Professor Paulo Donato Castellane, 14870-000 Jaboticabal, São Paulo, Brasil.

2) Departamento de Patologia Veterinária, Faculdade de Ciências Agrárias e Veterinárias, Universidade Estadual Paulista. Via de Acesso Professor Paulo Donato Castellane, 14870-000 Jaboticabal, São Paulo, Brasil.

3) E-mail: mtavares@caunesp.unesp.br. Bolsista CNPq. 
A relação hepatosomática também é influenciada pelo ciclo reprodutivo (AGostinHo et al. 1990; BABIERI et al. 1996) e pelo sexo (TVERANGER 1985; OLIVEIRA et al. 1997a). Isso ocorre devido a passagem de material do fígado para as gônadas na época da reprodução (LOVE 1970; BARBIERI \& AFONSO-MARINS 1995; BARBIERI et al. 1996). Outros fatores como alimentação e infecções também podem ser responsáveis por eventuais alterações em tais características biométricas em peixes teleósteos. RAFAEL \& BRAUNBECK (1988) ressaltam que peixes alimentados com ração artificial apresentaram maior ganho de peso corporal e de massa hepática, quando comparados aos que receberam somente alimentação natural. Como conseqüência há aumento do fator de condição e da relação hepatosomática nos peixes arraçoados.

A infecção experimental com Ichthyophthirius multifilis Fouquet, 1876, em carpas Cyprinus carpio Linnaeus, 1758 (Cyprinidae), reduziu o peso corporal, o hepático, o fator de condição e a relação hepatosomática, quando comparada ao grupo controle. E dependendo da intensidade da infecção houve aumento da relação esplenosomática nos peixes infectados (KUROVSKAYA \& OSADCHAYA 1993). Já esplenomegalia e hepatomegalia ocorrendo concomitantemente foi evidenciada em Salmo gairdneri Richardson, 1836 (Salmonidae) infectado experimentalmente com Cryptobia salmositica Katz, 1951 (LOWE-JINDE 1980).

A determinação do valor padrão médio do peso do baço e do figado, relação hepatosomática e esplenosomática e fator de condição são importantes para compreensão dos distúrbios que podem ocorrer durante os processos patológicos (QUENTEL \& OBACH 1992).

$\mathrm{Na}$ literatura são poucos os estudos referentes a tais variáveis biométricas em espécies brasileiras. Esses poucos estudos descrevem somente a relação hepatosomática em P. scrofa (VIEIRA 1984), C. macropomum (SAINT-PAUL 1984), Rhinelepis aspera Agassis, 1829 (Loricariidae) (AgostinHo et al. 1990), Astyanax bimaculatus Linnaeus, 1758 (Characidae) (BARBIERI \& AFONSO-MARINS 1995), Astyanax fasciatus Cuvier, 1819 (Characidae) (BARBIERI et al. 1996) e P. mesopotamicus (OLIVEIRA et al. 1997a).

O presente trabalho teve como objetivo descrever os valores médios do peso esplênico e hepático, relação esplenosomática (RSS), relação hepatosomática (RHS) e o fator relativo de condição (Kn) na tilápia Oreochromis niloticus Trewavas, 1983 (Cichlidae), pacu Piaractus mesopotamicus, piauçú Leporinus macrocephalus Garavelo \& Britski, 1988 (Anostomidae) e do híbrido tambacu (macho de P. mesopotamicus $\mathrm{x}$ fêmea de C. macropomum), sadios, mantidos em condições de cultivo.

\section{MATERIAL E MÉTODOS}

\section{Peixes e características de criação}

Cinqüenta espécimes de tilápia-do-Nilo Oreochromis niloticus (sexualmente revertidas), seis pacus Piaractus mesopotamicus, dez piauçus Leporinus macrocephalus e quinze híbridos de tambacu, cultivados intensivamente e alimentados com ração comercial foram colhidos de viveiro de $300 \mathrm{~m}^{2}$ em pesque-pague de 
Franca, São Paulo, Brasil (20³5'27" - 47'26'33"). Todos os peixes eram sexualmente imaturos e foram capturados com auxílio de caniço e anzol, e submetidos à avaliação biométrica e ao exame necroscópico.

\section{Avaliações biométricas}

De cada peixe foi medido o comprimento e o peso total. Em seguida, os peixes foram sacrificados por comoção cerebral para retirada e pesagem do baço e do fígado. Para tal utilizou-se balança digital com sensibilidade mínima de $0,01 \mathrm{~g}$ e máxima de $100 \mathrm{~g}$. De posse desses dados biométricos determinou-se:

1) Fator relativo de condição (Kn), de acordo com LE CREN (1951);

2) Relação hepatosomática (RHS\%): $\frac{\text { Peso do figado }(g)}{\text { Peso corporal }(g)} \times 100$

3) Relação esplenosomática (RSS\%): $\frac{\text { Peso do baço }(g)}{\text { Peso corporal }(g)} \times 100$

\section{Exame parasitológico}

Após a biometria, os peixes foram necropsiados segundo a técnica de rotina, investigando-se a eventual presença de alterações estruturais. Para pesquisa parasitológica foi colhido o muco da superfície corporal e fragmentos de brânquias, rins, fígado, baço e coração. Essas peças foram comprimidas entre lâmina e lamínula, com uma gota de solução de cloreto de sódio (0,65\%). Para procedimentos de identificação de parasitas utilizou-se as recomendações de MARTINS (1998) e PAVANELli et al. (1998).

\section{Análise da água dos viveiros}

No momento da colheita dos espécimes foram avaliados a temperatura da água com termômetro de bulbo, o pH em peagômetro eletrônico, a condutividade elétrica em condutivímetro e a concentração de oxigênio dissolvido em oxímetro.

\section{Análise estatística}

Os resultados encontrados para as diferentes variáveis foram analisados por regressão linear e teste $t$, ao nível de $5 \%$ de probabilidade (BEIGUELMAN 1996).

\section{RESULTADOS}

Ao exame necroscópico não foi observada a presença de parasitas ou de alterações estruturais.

A água apresentou as seguintes características: a temperatura variou de $25^{\circ}$ a $27^{\circ} \mathrm{C}$; o pH de 6,9 a 8,1 ; a condutividade de 31,0 a $78,0 \mu \mathrm{S} / \mathrm{cm}$ e o oxigênio dissolvido de 4,9 a $6,3 \mathrm{mg} / \mathrm{L}$.

Os resultados das variáveis biométricas determinadas estão expressos na tabela I. A $O$. niloticus apresentou relação hepatosomática maior, quando comparada ao $P$. mesopotamicus, $L$. macrocephalus e ao tambacu. Nesses três últimos a relação hepatosomática foi próxima. Já, a relação esplenosomática de $O$. niloticus e do L. macrocephalus foi similar, mas menores em relação ao $P$. mesopotamicus e tambacu. Esses últimos, por sua vez, apresentaram valores significativamente distintos entre si. 
Tabela I. Valores médios \pm desvio padrão das variáveis biométricas de 0 . niloticus, $P$. mesopotamicus, L. macrocephalus e tambacu. Os valores entre parênteses representam amplitudes de variação.

\begin{tabular}{|c|c|c|c|c|c|}
\hline \multirow[b]{2}{*}{ Peso Corporal (g) } & O. niloticus & P. mesopotamicus & L. macrocephalus & \multicolumn{2}{|c|}{ Tambacu } \\
\hline & $\begin{array}{c}420,000 \pm 7,700 \\
(320,000-740,000)\end{array}$ & $\begin{array}{c}560,000 \pm 105,800 \\
(480,000-680,000)\end{array}$ & $\begin{array}{r}514,000 \pm 268,100) \\
(270,000-870,000)\end{array}$ & $\begin{array}{r}494,600 \pm \\
(110,000-\end{array}$ & $\begin{array}{l} \pm 388,500 \\
-1330,000)\end{array}$ \\
\hline Comprimento $(\mathrm{cm})$ & $\begin{array}{c}28,200 \pm 1,900 \\
(25,000-34,500)\end{array}$ & $\begin{array}{cc}32,500 \pm & 4,300 \\
(30,000-37,500)\end{array}$ & $\begin{array}{c}32,700 \pm 6,400 \\
(27,000-41,000)\end{array}$ & $\begin{array}{r}28,400 \pm \\
(18,000-\end{array}$ & $\begin{array}{l} \pm \quad 8,200 \\
-\quad 42,500)\end{array}$ \\
\hline Peso Baço $(\mathrm{g})$ & $\begin{array}{cc}0,210 \pm & 0,160 \\
(0,100- & 0,400)\end{array}$ & $\begin{array}{cc}1,130 \pm & 0,210 \\
(0,900- & 1,300)\end{array}$ & $\begin{array}{cc}0,310 \pm & 0,210 \\
(0,100- & 0,700)\end{array}$ & $\begin{array}{r}0,420 \pm \\
(0,100-\end{array}$ & $\begin{array}{ll} \pm & 0,260 \\
-\quad 0,800)\end{array}$ \\
\hline Peso Figado (g) & $\begin{array}{c}6,100 \pm 1,600 \\
(2,800-10,600)\end{array}$ & $\begin{array}{c}6,000 \pm 0,700 \\
(5,200-5,900)\end{array}$ & $\begin{array}{r}5,100 \pm \\
(2,800-\end{array}$ & $\begin{array}{l}5,600 \pm \\
(0,900-\end{array}$ & $\begin{array}{c}4,600 \\
15,500)\end{array}$ \\
\hline RHS (\%) & $\begin{array}{c}1,440 \pm 0,280 \\
(0,870-1,790)\end{array}$ & $\begin{array}{c}1,050 \pm 0,140 \\
(0,980-1,230)\end{array}$ & $\begin{array}{r}1,070 \pm \\
(0,640-\end{array}$ & $\begin{array}{r}1,090 \pm \\
(0,750-\end{array}$ & $\begin{array}{l}0,220 \\
1,160)\end{array}$ \\
\hline RSS (\%) & $\begin{array}{c}0,052 \pm 0,017 \\
(0,024-0,114)\end{array}$ & $\begin{array}{c}0,198 \pm 0,040 \\
(0,173-0,250)\end{array}$ & $\begin{array}{c}0,056 \pm 0,017 \\
(0,027-0,080)\end{array}$ & $\begin{array}{r}0,132 \pm \\
(0,050-\end{array}$ & $\begin{array}{l}0,131 \\
0,454)\end{array}$ \\
\hline Fator de Condição & $\begin{array}{c}1,000 \pm 0,010 \\
(0,970-1,030)\end{array}$ & $\begin{array}{c}0,999 \pm 0,006 \\
(0,998-1,010)\end{array}$ & $\begin{array}{cc}1,004 \pm & 0,008 \\
(0,980 & -1,020)\end{array}$ & $\begin{array}{r}1,000 \pm \\
(0,960-\end{array}$ & $\begin{array}{l}0,030 \\
1,020)\end{array}$ \\
\hline
\end{tabular}

$\mathrm{O}$ estudo da regressão linear entre o peso hepático e o peso corporal, em cada espécie, mostrou correlação positiva. Admitindo a expressão y= A + Bx, em números não logaritmizados, onde y representa o peso hepático e x o peso corporal, a equação que permite estimar o peso hepático cujo peso corporal seja conhecido é: (a) O. niloticus (Fig. 1A) y=1,5302+0,0107x, sendo o $r=0,604, \mathrm{P}<0,01$; (b) $P$. mesopotamicus (Fig. 1B) $\mathrm{y}=2,7598+0,0057 \mathrm{x}, r=0,811, \mathrm{P}=0,05$; (c) L. macrocephalus (Fig. 1C) $\mathrm{y}=2,4923+0,0052 \mathrm{x}, r=0,737, \mathrm{P}<0,05$; (d) tambacu (Fig. 1D) $\mathrm{y}=$ $-0,2027+0,0117 \mathrm{x}, r=0,983, \mathrm{P}<0,01)$.

A análise de correlação linear entre o peso do fígado e o comprimento corporal total do peixe evidenciou correlação positiva em todas as espécies estudadas. Admitindo a expressão $\mathrm{y}=\mathrm{A}+\mathrm{Bx}$, em números não logaritmizados, onde $\mathrm{y}$ representa o peso hepático $\mathrm{e} \times \mathrm{o}$ comprimento corporal, a equação que permite estimar o peso hepático cujo comprimento corporal seja conhecido é: (a) O. niloticus (Fig. 2A) $\mathrm{y}=-7,674+0,4876 \mathrm{x}$, s endo o $r=0,560, \mathrm{P}<0,01$; (b) $P$. mesopotamicus (Fig. 2B) $\mathrm{y}=0,9118+0,1558 \mathrm{x}, r=0,888, \mathrm{P}<0,05$; (c) L. macrocephalus (Fig. $2 \mathrm{C}$ ) $y=-1,9423+0,2171 x, r=0,737, P<0,05$; (d) tambacu (Fig. 2D) $y=-9,5719+$ $0,5327 \mathrm{x}, r=0,951, \mathrm{P}<0,01$.

A correlação entre o peso do baço e o peso corporal em $O$. niloticus, $P$. mesopotamicus e tambacu não foi estatisticamente significativa. Já em L. macrocephalus essas correlações foram altamente significativa. Admitindo a expressão $\mathrm{y}=$ $\mathrm{A}+\mathrm{Bx}$, em números não logaritmizados, onde y representa o peso esplênico e x 0 peso corporal, as respectivas equações obtidas foram: (a) $O$. niloticus (Fig. $3 \mathrm{~A}$ ) $\mathrm{y}=$ $0,1534+0,0001 \mathrm{x}$, sendo o $r=0,185, \mathrm{P}>0,05$; (b) P. mesopotamicus (Fig. 3B) $\mathrm{y}=$ $0,5458+0,001 \mathrm{x}, r=0,554, \mathrm{P}>0,05$; (c) L. macrocephalus (Fig. $3 \mathrm{C}$ ) $\mathrm{y}=-0,1867+$ $0,001 \mathrm{x}, r=0,980, \mathrm{P}<0,01$; (d) tambacu (Fig. 3D) $\mathrm{y}=0,2995+0,0002 \mathrm{x}, r=0,366$, $\mathrm{P}>0,05$.

A correlação linear entre o peso do baço e o comprimento corporal total em O. niloticus, $P$. mesopotamicus e tambacu não foi estatisticamente significativa. Porém, em L. macrocephalus essa correlação foi altamente significativa. Admitindo a expressão $\mathrm{y}=\mathrm{A}+\mathrm{Bx}$, em números não logaritmizados, onde y representa o peso 
esplênico e x o comprimento corporal, obteve as seguintes equações: (a) $O$. niloticus (Fig. 4A) $\mathrm{y}=-0,0144+0,008 \mathrm{x}$, sendo o $r=0,242, \mathrm{P}>0,05$; (b) P. mesopotamicus (Fig. 4B) $\mathrm{y}=0,1053+0,0311 \mathrm{x}, r=0,681, \mathrm{P}>0,05$; (c) L. macrocephalus (Fig. 4C) $y=-1,0176+0,0409 x, r=0,974, P<0,01 ;$ (d) tambacu (Fig. 4D) $y=0,0795+0,0121 x$, $r=0,375, \mathrm{P}>0,05$.
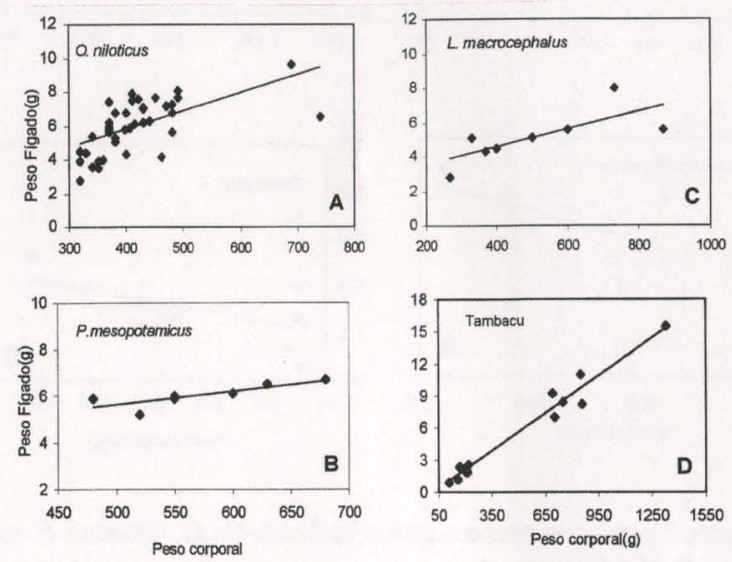

Fig. 1. Relação entre o peso corporal e o peso hepático em O. niloticus, $P$. mesopotamicus, L. macrocephalus e tambacu.
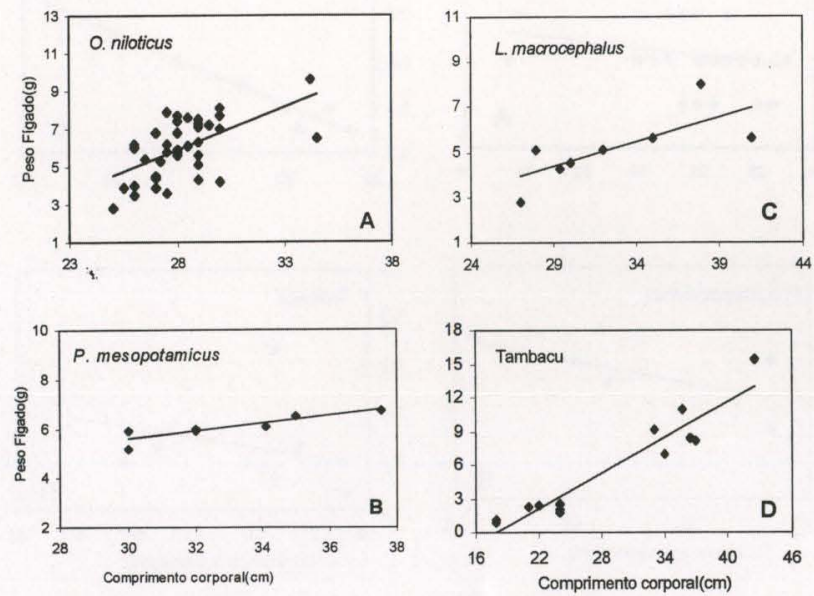

Fig. 2. Relação entre o comprimento corporal e o peso hepático em O. niloticus, $P$. mesopotamicus, L. macrocephalus e tambacu. 

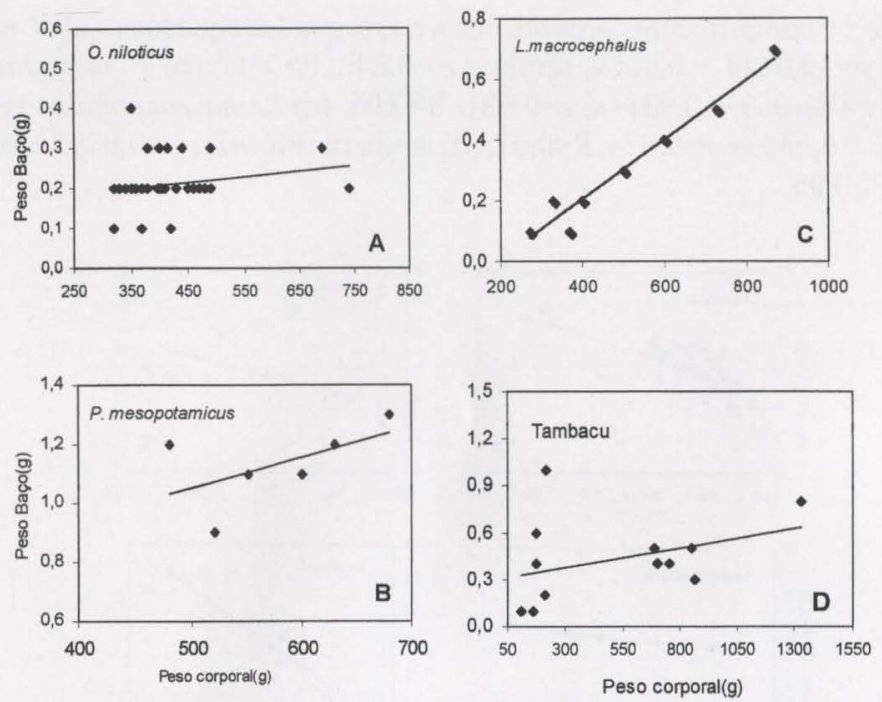

Fig. 3. Relação entre o peso corporal e o peso esplênico em O. niloticus, $P$. mesopotamicus, L. macrocephalus e tambacu
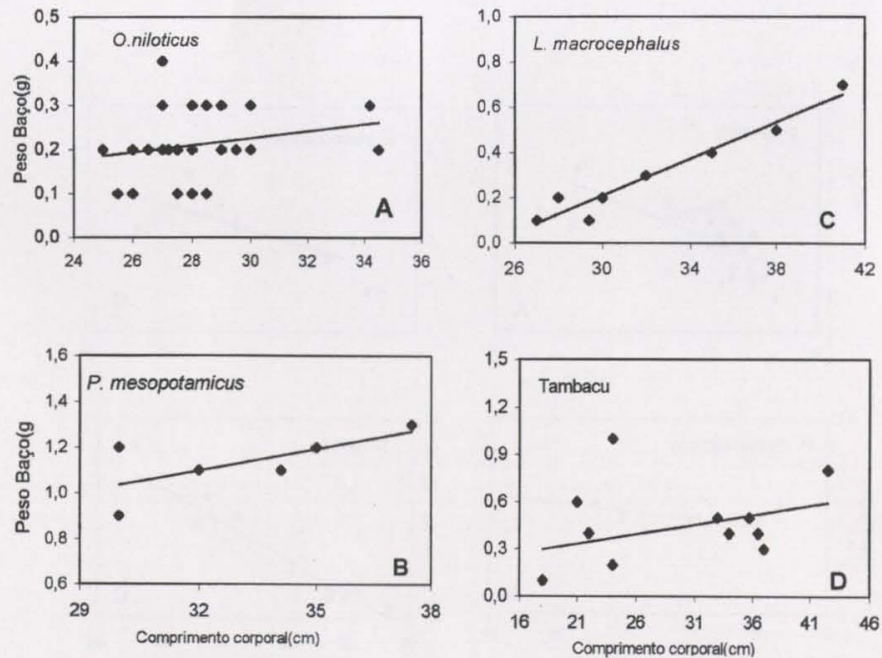

Fig. 4. Relação entre o comprimento corporal e o peso esplênico em $O$. niloticus, $P$. mesopotamicus, L. macrocephalus e tambacu.

Revta bras. Zool. 17 (1): 273 - 281, 2000 


\section{DISCUSSÃO}

De acordo com QUENTEL \& OBACH (1992) a determinação dos valores padrão para a relação hepatossomática e esplenosomática é importante para a compreensão dos distúrbios hepáticos e esplênicos, que podem ocorrer durante os processos patológicos. No presente trabalho, o elevado valor da relação hepatosomática de $O$. niloticus demonstra que o fígado dessa espécie é relativamente maior que o observado no $P$. mesopotamicus, no $L$. macrocephalus e tambacu. O fígado de $O$. niloticus também é maior que o de outros peixes como o $P$. scrofa (VIEIRA 1984), R. aspera (AgostinHo et al. 1990), Scophthalmus maximus Linnaeus, 1758 (QUENTEL \& OBACH 1992) e A. fasciatus (BARBIERI et al. 1996). Por outro lado, o valor da relação hepatosomática de $O$. niloticus é baixo se comparado com juvenis de O. mossambicus Peters, 1852 (Cichlidae) (Dioundick \& STOM 1990) e com o híbrido O. niloticus x O. aureus (SHIAU \& HWANG 1993; SHIAU \& LUNG 1993). Portanto, o fígado de $O$. niloticus é menor que o de outras tilápias.

Os valores médios da relação hepatosomática do $P$. mesopotamicus, determinados neste trabalho, são menores que os descritos para juvenis de C. macropomum do Lago Mandaraqui, no Amazonas (SAINT-PAUL 1984). Porém, foram superiores aos das fêmeas de $P$. mesopotamicus e próximos aos dos machos, descritos por Oliveira et al. (1997a). Contudo, esses autores trabalharam com $P$. mesopotamicus maduros, enquanto neste trabalho os peixes eram imaturos. Já SAINT-PAUL (1984) cita que durante o período de cheia na região amazônica, o $C$. macropomum ingere sementes que estão à disposição, aumentando assim o glicogênio hepático e a relação hepatosomática. Esse estoque serve de energia para o período de estiagem, quando ocorre redução da oferta de alimentos e conseqüentemente de sua relação hepatosomática.

Similarmente, níveis elevados de carboidratos na dieta de truta $S$. gairdneri resultaram em aumento do glicogênio hepático e do peso do fígado (HILTON 1982). RAFAEL \& BRAUNBECK (1988) observaram maior ganho de peso corporal e de massa hepática nos peixes mantidos com ração artificial, quando comparado com peixes mantidos com alimentação natural. Então o arraçoamento aumenta a relação hepatossomática e o fator de condição. Esse fator permite comparar duas ou mais espécies vivendo em diferentes condições de alimentação, densidade populacional, clima e outras variáveis. Além disso, pode ser empregado para acompanhar o grau de atividade alimentar de uma dada espécie (WEATHERLEY \& GILL 1987).

Os valores médios da relação esplenosomática de $O$. niloticus e $L$. macrocephalus foram similares. Porém, são inferiores aos de $P$. mesopotamicus e tambacu. Esses últimos, por sua vez, apresentaram valores distintos entre si, sendo maior no híbrido tambacu ( $P$. mesopotamicus x C. macropomum). L. macrocephalus e $O$. niloticus apresentaram valores médios de peso esplênico e relação esplenosomática superiores aos descritos por QUENTEL \& OBACH (1992) em S. maximus. Em contrapartida, o baço do L. macrocephalus e $O$. niloticus é menor que do Dicentrarchus labrax Linnaeus, 1758 (HADJKACEM et al. 1986; QUENTEL \& OBACH 1990) e do S. gairdneri (TATNER \& MANNING 1983). Já o baço do P. mesopotamicus é relativamente maior que dessas espécies. Todavia, tais valores além de certa especificidade podem variar com a idade, estado fisiológico do animal, com o 
ambiente (QUENTEL \& OBACH 1992), com o ciclo reprodutivo (AGOSTINHO et al. 1990; BARBIERI et al. 1996), com o sexo (TVERANGER 1985; OlIVEIRA et al. 1997a) e em situações de estresse (PETERS \& SCHWARZER 1985).

A análise de regressão linear demonstrou correlação positiva entre o peso hepático e o peso corporal, assim como entre o peso hepático e o comprimento total em $O$. niloticus, $L$. macrocephalus, $P$. mesopotamicus e tambacu, à semelhança do observado em Salvelinus fontinalis Mitchill, 1814 (Salmonidae) (LARSON 1973).

Os resultados demonstraram que não houve correlação significativa entre o peso corporal e o peso esplênico, assim como entre o comprimento e peso esplênico. Em contrapartida, YAMAMOTO \& SHIMA (1990) demonstraram correlação positiva entre o peso corporal e o peso esplênico em $O$. niloticus.

O baço é um importante órgão eritro e leucopoético (QUENTEL \& OBACH 1992). Esplenomegalia sugere o desenvolvimento de respostas leucocitárias às infecções ou à produção de eritrócitos para reposição sangüínea em casos de processos anemiantes. O aumento do volume do baço ocorre devido a alterações bioquímicas e fisiológicas necessárias para manter a homeostasia orgânica como resposta a infecções (LOWE-JINDE 1980) ou outro tipo de agressão ambiental.

\section{CONCLUSÕES}

Oreochromis niloticus apresentou maior RHS do que o P. mesopotamicus, L. macrocephalus e tambacu. Porém, nesses três últimos, o RHS foi bem próximo. O RSS na $O$. niloticus e no L. macrocephalus foram similares entre si, mas inferiores ao $P$. mesopotamicus e tambacu. Esses, por sua vez, apresentaram valores distintos entre si. A relação peso hepático/peso corporal e peso hepático/ comprimento corporal mostrou correlação positiva em todos os peixes estudados. A relação peso esplênico/peso corporal e peso esplênico/comprimento corporal não foram significativas $(\mathrm{P}>0,05)$ em $O$. niloticus, $P$. mesopotamicus e tambacu, mas altamente significativas no L. macrocephalus $(\mathrm{P}<0,01)$.

\section{REFERÊNCIAS BIBLIOGRÁFICAS}

Agostinho, A.A.; G. BARBieri; J.R. Verani \& N.S. HaHn. 1990. Variação do fator de condição e do indice hepatossomático e seus relações com o ciclo reprodutivo em Rhinelepis aspera (Agassis, 1829) (Osteichthyes, Loricariidae) no rio Paranapanema, Porecatu, Pr. Ciência e Cultura 42 (9): 711-714.

Beiguelman, B. 1996. Curso prático de bioestatística. Ribeirão Preto, Sociedade Brasileira de Genética, 242p.

BARBIERI, G. \& M. Afonso-Marins. 1995. Estudo da dinâmica da reprodução de fềmeas de Astyanax bimaculatus (Linnaeus, 1758) da represa do Lobo, Estado de São Paulo (Osteichthyes, Characidae). Arq. Biol. Tecnol. 38 (4): 1191-1197.

BARBIERI, G.; S.M. HARTZ \& J.R. Verani. 1996. O fator de condição e índice hepatossomático como indicadores do período de desova de Astyanax fasciatus da represa do Lobo, Estado de São Paulo (Osteichthyes, Characidae). Iheringia 81: 97-100.

Dioundick, O. B. \& D.I. Sтом.1990. Effects of dietary $\alpha$-cellulose levels on the juvenile tilapia, Oreochromis mossambicus (Peters). Aquaculture 91: 311-315.

HADJKACEM, N.; J.F. AldRIN \& B. RomestAND. 1986. Influence immediate du prossage des bacs sur certains parametres sanguins du loup d'elevage, Dicentratrarchus labrax L. Effet de estres. Aquaculture 59 (1): 53-59. 
HEIDINGER, R.C. \& S.D. CRAWFORD. 1977. Effect of temperature and feeding rate on the liver-somatic index of the Largemouth Bass, Micropterus salmoides. Jour. Fish Res. Board 34: 633-638.

HiLton, J.W. 1982. The effect of pre-fasting diet and water temperatura on liver glycogen and liver weigth in rainbow trout, Salmo gairdneri Richardson, during fasting. Jour. Fish Biol. 20: 69-78.

HoAR,W.S. \& D.J. Randall. 1971. Fish physiology. New York, Academic Press Inc., 457p.

KUROSVSKAYA, L.N. \& S.A. OSADCHAYA. 1993. The influence of Ichthyophthirius multifiliis on underyearling carp, Cyprinus carpio. Jour. Ichthyol. 33 (4): 81-92.

LARSON, G.L. 1973. Liver weigth of Brook Trout in a high-mountain lake in Washington State. Prog. Fish-Culturist 35 (4): 234-236.

LE CREN, E.D. 1951. The length-weight relationship and seasonal cycle in gonadal weight and condition in the perch (Perca fluviatilis). Jour. Anim. Ecol. 20 (2): 201-219.

Love, R.M. 1970. The chemical biology of fish. New York, Academic Press, 547p.

LOWE-JINDE, L. 1980. Observations of rainbow trout, Salmo gairdneri Richardson, infected with Cryptobia salmostica. Jour. Fish Biol. 17 (1): 23-30.

Martins, M.L. 1998. Doenças infecciosas e parasitárias de peixes. Jaboticabal, Funep, 65p.

Oliveira, E.G.; E.C. Urbinati; V.L. Souza \& D.P. Roviero. 1997a. Concentração de glicogênio em diferentes tecidos de pacu (Piaractus mesopotamicus), Holmberg, 1887. Bol. Inst. Pesca 24 (especial): 89-95.

-1997b. Índice gordura-visceral-somática e níveis de lipídio em diferentes tecidos de pacu (Piaractus mesopotamicus, Holmberg, 1887). Bol. Inst. Pesca 24 (especial): 97-103.

PavANElli, G.C.; J.C. EirAS \& R.M. TAKEMOTO. 1998. Doenças de peixes: profilaxia, diagnóstico e tratamento. Maringá, Ed. Eduem, 259p.

PETERS, G. \& R. SCHWARZER. 1985. Chages in hematopoietic tissue of rainbow trout under influence of stress. Dis. Aquat. Organ. 1: 1-10.

Quentel C. \& A. OBACH. 1990. La composition cellulaire des organes hématopoétiques du bar, Dicentratrarchus labrax L. Ichtyophysiol. Acta 13: 59-70.

- 1992. The cellular composition of the blood and haematopoietic organs of turbot Scophthalmus maximus L.. Jour. Fish Biol. 41: 709-716.

RAFAEL, J. \& T. BRAUNBECK. 1988. Interacting effects of diet and environmental temperature on biochemical parameters in the liver of Leuciscus idus melanotus (Cyprinidae: Teleostei). Fish Physiol. Biochem. 5 (1): 9-19.

SAINT-PAUL, U. 1984. Investigations on the seasonal changes in the chemical composition of liver and condition from a neotropical characoid fish Colossoma macropomum (Serrasalmidae). Amazoniana 9 (1): 147-158.

SHIAU, S.Y. \& C.Q. Lung. 1993. No dietary vitamin $B_{12}$ required for juvenile tilapia Oreochromis niloticus $\mathrm{x} O$. aureus. Comp. Biochem. Physiol 105A: 147-150.

SHIAU, S.Y. \& J.Y. HWANG.1993. Vitamin D requirements of juvenile hybrid tilapia Oreochromis niloticus x $O$. aureus. Nippon Suisan Gakkaishi 59 (3): 553-558.

TATNER, M.F. \& M.J. MANNING. 1983. Growth of the lymphoid organs in rainbow trout, Salmo gairdneri from one to fifteen months age. Jour. Zool. 199: 503-520.

TVERANGER, B. 1985. Variation in growth rate, liver weigth and body composition at first sexual maturity in rainbow trout. Aquaculture 49 (2): 89-99.

VieIRA, A.L. 1984. Aspectos do metabolismo lipídico do curimbatá Prochilodus scrofa (Steindachner, 1881) no estádio de repouso gonadal. Bol. Inst. Pesca 11: 63-68.

WEATHERLEY, A.H. \& H.S. Gill. 1987. The biology of fish growth. New York, Academic Press, 429p.

Yамамото, K.I. \& T. Sнiма. 1990. Relationship of spleen to body weight before and after contraction in a teleost fish Oreochromis niloticus. Comp. Biochem. Physiol 96A (1): 107-108.

Recebido em 01.VII.1999; aceito em 03.III.2000. 